\title{
Ablation Threshold Measurement and Chemical Modification of UV Nanosecond Laser Micromachining of Polycrystalline Diamond
}

Naveed Ullah ( $\square$ naveed01@stu.xjtu.edu.cn )

Xi'an Jiaotong University

Jianlei Cui

Xi'an Jiaotong University

Zhengjie Fan

Xi'an Jiaotong University

Xuesong Mei

Xi'an Jiaotong University

\section{Research Article}

Keywords: Ablation threshold, Chemical modification, UV nanosecond laser, PCD diamond

Posted Date: February 15th, 2021

DOI: https://doi.org/10.21203/rs.3.rs-178399/v1

License: (a) (i) This work is licensed under a Creative Commons Attribution 4.0 International License.

Read Full License

Version of Record: A version of this preprint was published at physica status solidi (a) on October 31st, 2021. See the published version at https://doi.org/10.1002/pssa.202100450. 


\title{
Ablation Threshold Measurement and Chemical Modification of UV Nanosecond Laser Micromachining of Polycrystalline Diamond Naveed Ullah", ${ }^{\mathrm{a}, \mathrm{b}}$, Jianlei Cui ${ }^{\mathrm{a}, \mathrm{b}, \mathrm{c}}$, Zhengjie Fan ${ }^{\mathrm{a}, \mathrm{b}, \mathrm{c}}$, Xuesong Mei ${ }^{\mathrm{a}, \mathrm{b}}$
}

${ }^{a}$ State Key Laboratory for Manufacturing Systems Engineering, Xi'an Jiaotong University, Xi'an 710049, P.R. China

\author{
${ }^{\mathrm{b}}$ Shaanxi Key Laboratory of Intelligent Robots, Xi'an Jiaotong University, Xi'an 710049, P. R. \\ China \\ c Xi’an Jiaotong University Shenzhen Research Institute, Shenzhen 518057, P. R. China
}

Corresponding Author: naveed01@stu.xjtu.edu.cn

\begin{abstract}
In recent years, the influence of pulsed laser interaction with material has become a research hotspot. The micromachining of polycrystalline diamond with pulsed laser is a very important research direction. This study scrutinized the ablation threshold and chemical modification of PCD irradiated by UV nanosecond laser, which were not investigated before. Irradiated a bulk PCD in air using a UV nanosecond laser source to perform multi-pulse processing at a wavelength of $355 \mathrm{~nm}$. Based on the linear relation between the laser pulse energy density with crater diameter the Gaussian distribution of the laser intensity on the cross-section the ablation threshold of PCD was determined to be about $3.7326 \mathrm{~J} / \mathrm{cm}^{2}$. And also investigated that the photochemical processing of UV nanosecond laser irradiation of $\mathrm{PCD}$ the $\mathrm{sp}^{3}$ structure transforms to the $\mathrm{sp}^{2}$ graphite phase. So, this study contributes a theoretical and experimental reference for the UV nanosecond laser micromachining of PCD material in research and manufacturing industries.
\end{abstract}

Keywords: Ablation threshold; Chemical modification; UV nanosecond laser; PCD diamond 


\section{Introduction}

Diamond is the extremely hardest material with randomly orientated diamond particles in a metal matrix, has record value thermal conductivity at room temperature, high hardness, sound velocity, and carrier mobility ${ }^{[1]}$. It is widely used in aerospace, military, electronics, precision machinery, as a cutting tool material ${ }^{[2]}$ for a variety of industrial applications and other fields. Because of the extremely high Mohs hardness of diamonds ${ }^{[3]}$, it is very hard for traditional machining to realize its precise machining. On the contrary, laser as a unique kind of non-contact processing method with high peak power, more limited thermal damage, high precision processing, and specific processing range can cut PCD and other hard material efficiently ${ }^{[4-7]}$. With the evolution of laser micromachining technology, particularly pulse lasers have earned much more consideration although there are some constraints ${ }^{[8,9]}$. So pulsed laser ablation is one of the most famous manufacturing methods which is widely used for precision machining or in semiconductor industries. Currently, several researchers have studied the laser processing of diamond. Xing et al. ${ }^{[10]}$ used a nanosecond pulsed laser to process micro-groove on the surface of a polycrystalline diamond. They found that the height of the micro-groove increases with increasing laser power and decreasing with scanning speed and repetition rate. Ren. J et al. ${ }^{[1]]}$ suited, the accuracy of nanosecond laser ablation for general precision machining, and Compared to ultrashort laser ablation, nanosecond laser ablation is more efficient and consequently more suitable for general precision machining processes.

Additionally, diamonds undergo phase transitions due to laser irradiation. The laser ablation of diamond takes place via surface graphitization due to a combined thermal and photochemical effect ${ }^{[12]}$. G. Eberle et al. ${ }^{[13]}$ were processed a good quality diamond and polycrystalline diamond by different laser action, and studied the thermal effects of different pulse width laser processing on the polycrystalline diamond and analyzed ablative mechanism qualitatively and quantitatively. Kononenko ${ }^{[14]}$ illustrated the diamond graphitization phase change model system to determine the laser parameters that influence the graphitization of diamond. Chen ${ }^{[15]}$ elaborated the phase transition model, summarized the graphitization and material ablation mechanism of pulsed laser on the micromachining diamond material. Still, there is no precise experimental research status is not well studied to verify the graphitization of polycrystalline diamond, and if there is experimental support, it will provide a great guide for UV nanosecond laser processing of diamond. Therefore, this paper is based on the research of laser microfabrication on polycrystalline diamond mechanism and combined with experiments to reveal the internal chemical modification of PCD before and after UV nanosecond laser processing. 


\section{Materials and Methods}

In this experiment, the UV nanosecond laser micro/nano processing system INNO FOTIA355-OEM-2 was utilized, to investigate the diamond graphitized phase transitions and micro/nanostructures. The core wavelength of the laser from this device has a $355 \mathrm{~nm}$, a pulse width of $10 \mathrm{~ns}$, a repetition frequency range of $40-150 \mathrm{kHz}$, an average maximum power of $10 \mathrm{~W}$, and a quality factor of $\mathrm{M}^{2}=1.11$. The pictorial view of the UV nanosecond laser processing system is shown in Figure 1. The laser beamed from the laser device passed through the beam expander, the aperture, serval mirror lens, and lastly entered into the two-dimensional scanning galvanometer, which can focus the laser light and control to transfer on the sample surface. The beam expander was used here to settle the divergence angle of the laser. Aperture can enhance the laser spot shape.

The diamond sample used in the experiment was polycrystalline diamond and processing size of $10 \times 5 \times 2 \mathrm{~mm}$. Before processing, the sample was ultrasonically clean with acetone, absolute ethyl alcohol, and deionized water for 10 to 15 minutes. To remove dust particles and feculence from the surface and then air-dried. Afterward, fixed the sample on the twodimensional mobile platform to be processed. Describe the laser scanning path in scan master design software to control the scanning galvanometer to move the laser as an expected route. High-quality processing of PCD material to achieve for the various application. The sample was cleaned again with the same method to remove the processing residues after processing. Then finally, the cutting morphology was identified, and analyze through X-ray photoelectron spectroscopy (XPS) before and after laser treatment to comprehend out the internal chemical modification means phase transition induced by the UV nanosecond laser.

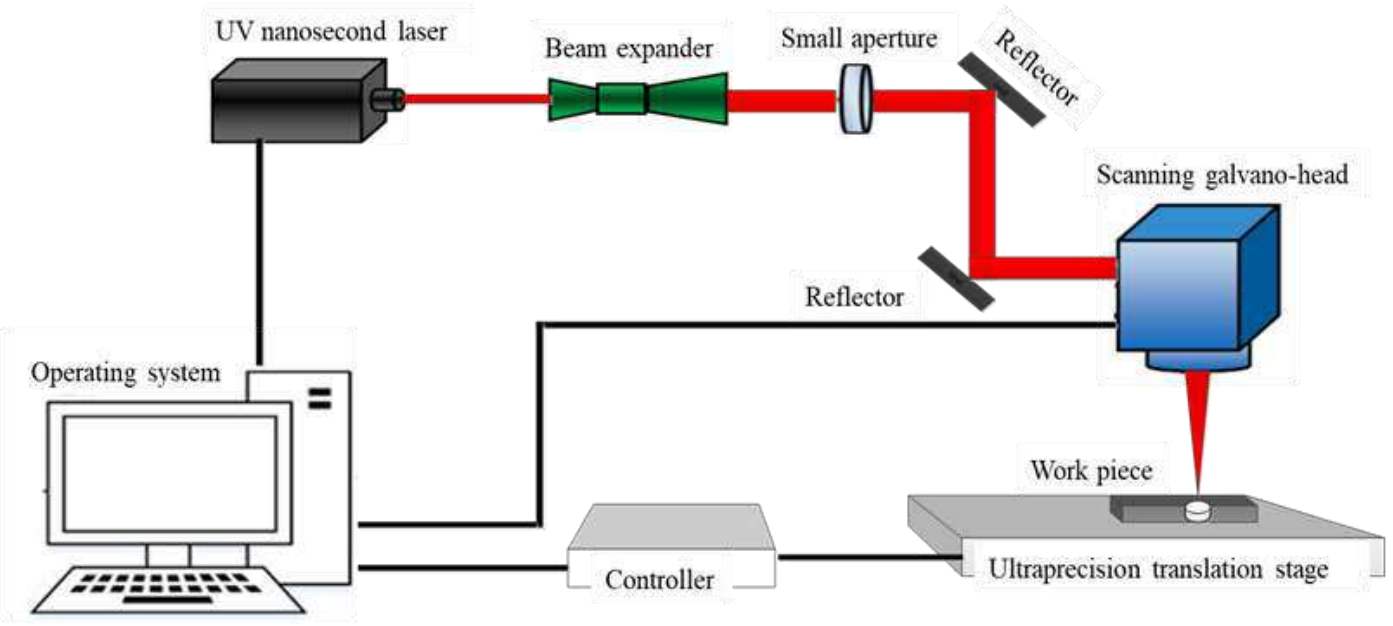

Figure 1 Schematic diagram of UV nanosecond laser machining system 


\section{Results and discussion}

\section{1) Mathematical modeling of ablation threshold}

When the nanosecond laser is used to process the machining of a polycrystalline diamond, only the energy of the light spot is increase to etching of the material, the material will be removed. So, the ablation threshold is the basic and inherent parameter and minimum laser energy/ fluence of a laser source. Threshold theory ${ }^{[16]}$ asserts that the ablation threshold of the pulsed laser has a very small deviation, and the ablation threshold is the lowest laser energy density when the materials can be damaged by the laser ${ }^{[17,18]}$. Meanwhile, the nanosecond laser interacts with the processed sample in the form of pulses, the diamond surface interacts with the ablation to induce the chemical modification of the processed material, so the needed lowest power of the nanosecond laser in the processing process requires to be higher than the ablation threshold. It is necessary for producing ablation on the surface of a material. The light energy of nanosecond laser is Gaussian is shown in Fig 2, and the energy density at the center of the focus is high peak energy density $\left(\mathrm{F}_{0}^{\mathrm{Pk}}\right)$. The peak value of nanosecond laser energy density with single pulse energy $\left(\mathrm{E}_{\mathrm{p}}\right)$ relation is described below.

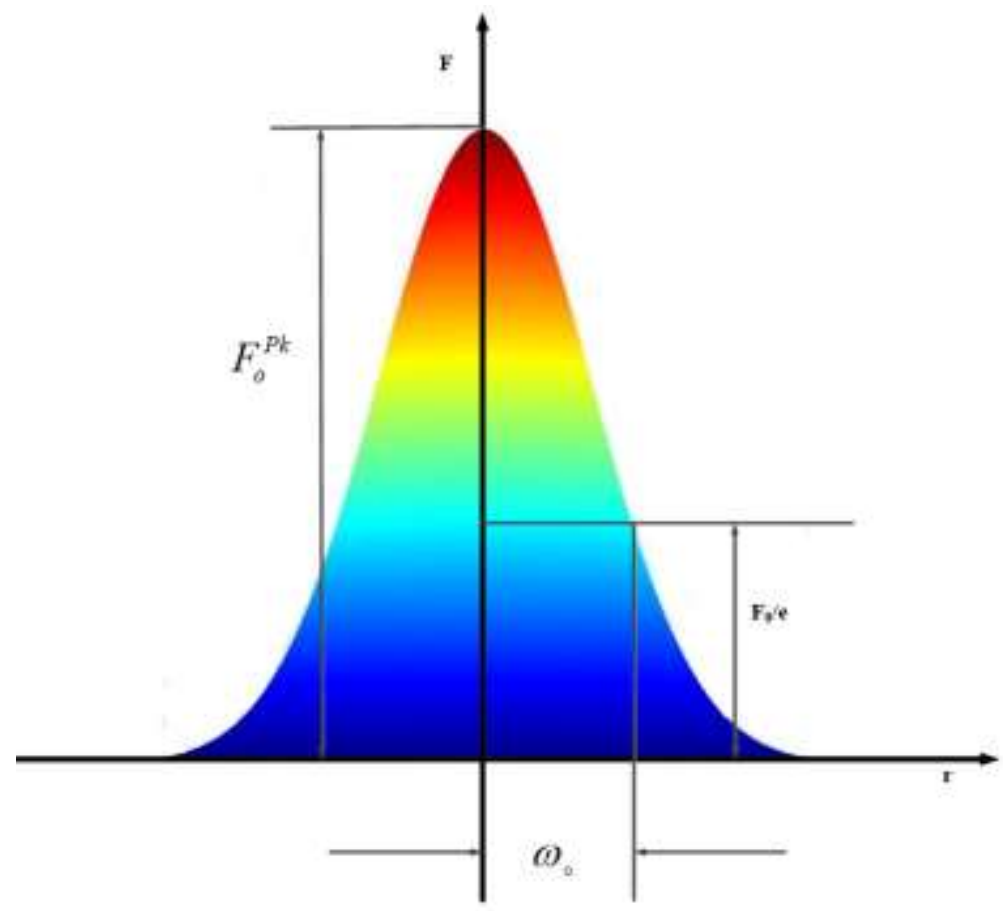

Figure 2. Energy distribution of gaussian beam 


$$
\begin{array}{r}
\mathrm{F}(\mathrm{r})=\mathrm{F}_{\mathrm{o}}^{\mathrm{Pk}} \times \exp \left(-2 \mathrm{r}^{2} / \omega_{\mathrm{o}}^{2}\right) \\
\mathrm{D}^{2}=2 \omega_{\mathrm{o}}^{2} \ln \left(\frac{2 \mathrm{E}_{\mathrm{P}} / \pi \omega_{\mathrm{o}}^{2}}{\mathrm{~F}_{\mathrm{th}}}\right)=2 \omega_{\mathrm{o}}^{2} \ln \mathrm{E}_{\mathrm{P}}+2 \omega_{\mathrm{o}}^{2} \ln \left(2 / \pi \omega_{\mathrm{o}}^{2} \mathrm{~F}_{\mathrm{th}}\right)
\end{array}
$$

$\mathrm{D}^{2}$ is the square of the micropore diameter, $\omega_{\mathrm{o}}$ is the laser beam waist radius, $\mathrm{F}_{\mathrm{th}}$ is the ablation threshold of the material, and $E_{p}$ is the single pulse energy. Based on the above mathematical analysis, the logarithmic value of laser single pulse energy and square value of the etching micropore satisfy linear relation. Therefore, the same processing condition laser beam spot radius after the nanosecond laser focusing is equal. Moreover, the ablation threshold of the material is constant value under nanosecond laser, the second term in equation 1-2 is constant. Micro-hole diameter obtained by nanosecond laser ablation at single and multi-pulse energies, measured experimentally and calculated which can be used to calculate the ablation threshold of PCD material.

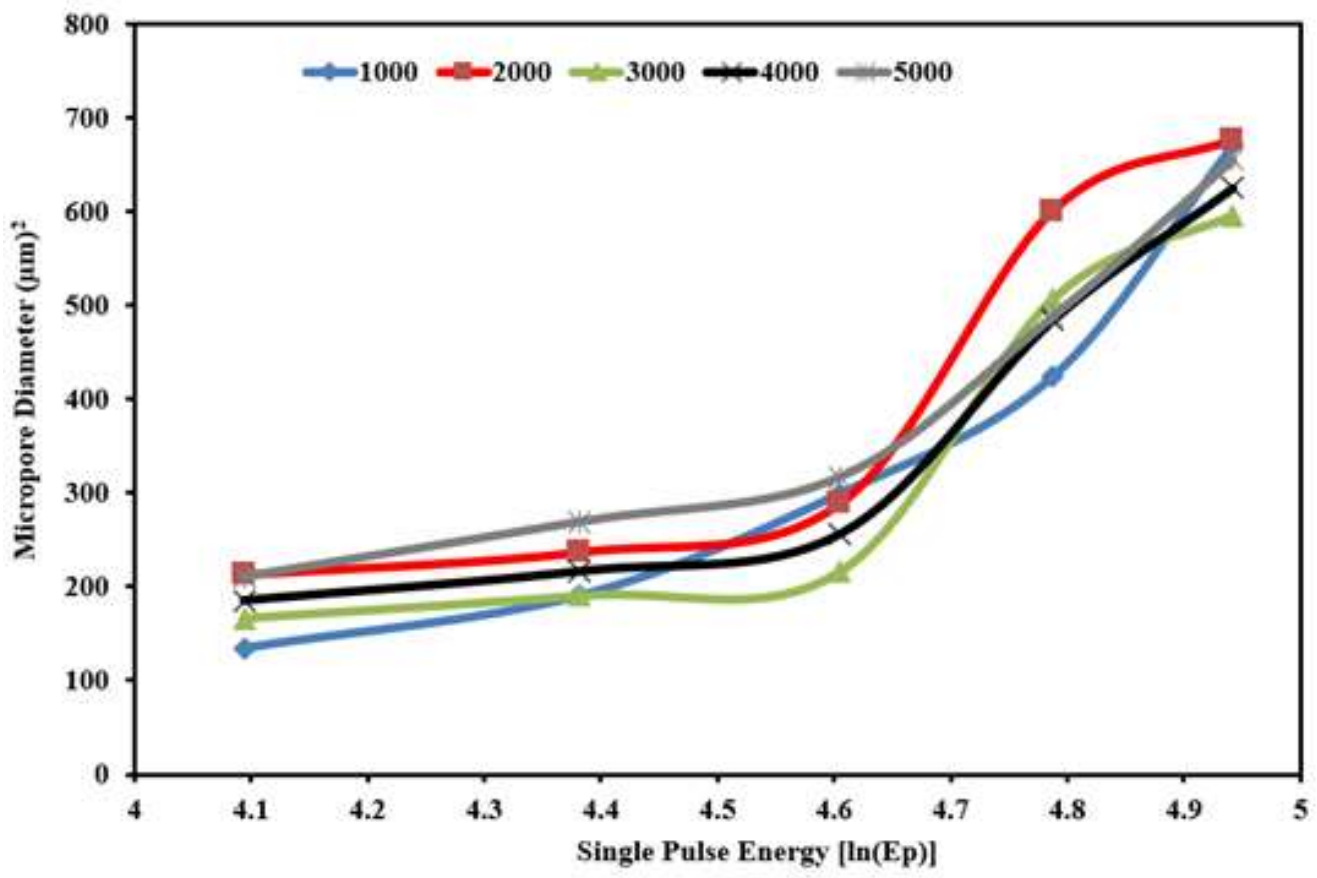

Figure $3 \ln \left(\mathrm{E}_{\mathrm{P}}\right)$ and square of micropore diameter at numbers of pulse.

To investigate the interaction between the nanosecond laser and the diamond during the ablation processing, it is essential to determine the ablation threshold of the diamond under the laser. Following the same processing conditions, the waist radius and material ablation threshold 
after the nanosecond laser focusing are fixed values, and the established linear correlation between single pulse energy and machined micropore is based on the equation 1-2. Measured the square diameter of each micropore and calculated the single pulse energy at different numbers of pulse was plotted as shown in Fig 3. We said that the square of the micropore diameter increases with different numbers of pulses at a single pulse power density of UV nanosecond laser. At the same time calculate the radius of the etched micropore and the square of the micropore diameter and single pulse laser power density under each number of pulses was calculated. We concluded that the laser beam spot radius value of each number of pulses is close to the laser beam spot radius, which can be a certain value as considered. So, high energy of laser and multiple pulses are used, the laser energy required for ablative materials will be lower than the ablation threshold when single pulse energy is used ${ }^{[19]}$. This phenomenon can be described by the logarithmic relationship between the cumulative number of pulses and the cumulative etching threshold for the number of pulses can be obtained.

$$
\begin{gathered}
\mathrm{F}_{\text {th }}(\mathrm{N})=\mathrm{F}_{\text {th }}(1) \times \mathrm{N}^{\mathrm{S}-1} \\
\ln \left[\mathrm{N} \times \mathrm{F}_{\text {th }}(\mathrm{N})\right]=\mathrm{S} \times \ln (\mathrm{N})+\ln \left[\mathrm{F}_{\mathrm{th}}(1)\right]
\end{gathered}
$$

The logarithm of the $\mathrm{N}$ number of pulses and the logarithm relation of the ablation threshold was fitted linearly as shown in Fig 4. Under the cumulative effect of the number of pulses, the single pulse ablation threshold value was obtained $F_{\text {th }}(1)=3.7326 \mathrm{~J} / \mathrm{cm}^{2}$ with corresponding cumulative coefficient $S=0.985$. So, verified that the measured value of the ablation threshold under the number of pulses was accurate and reliable. Determined that the ablation threshold of single-pulse laser energy density is higher than the number of pulses. It's necessary to use single pulse laser energy for permanent removal of material and get micro-hole with high depth. 


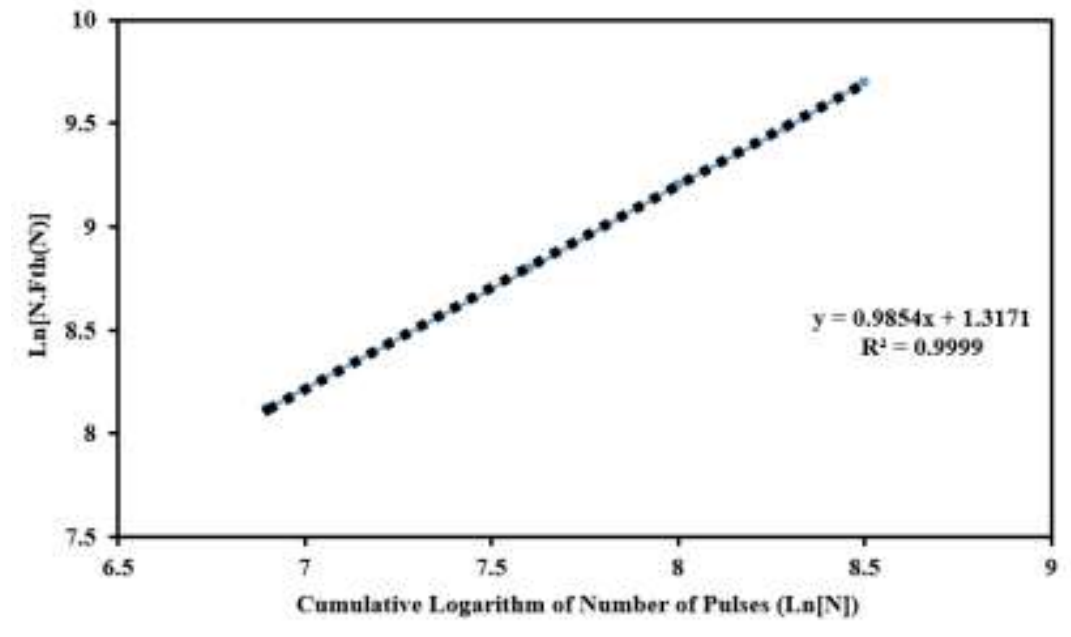

Figure 4 Cumulative effect of the number of pulses.

\section{2) Chemical modification}

The diamond crystal belongs to the cubic crystal system, which has a face-centered cubic (FCC) cell structure; the other four carbons form covalent bonds. At present, it is commonly used in diamond material characterization techniques, which has an important feature of the $\mathrm{X}$ ray photoelectron spectrometer analysis technique is not only the possibility to identify chemical elements but also to perform a quantitative analysis of the material. The ablation process of laser interacting with material starts with internal structure means the electrons of the material ${ }^{[20]}$. Additionally, at the time of laser irradiation of polycrystalline diamond, the cause why graphitization occurs during polycrystalline diamond machining is that the electrons of the surface absorb energy with the assistance of inverse bremsstrahlung ${ }^{[21]}$. The atomic absorption energy of $\mathrm{sp}^{3}$ in the diamond structure attains the transition energy and bounce to the binding state of $\mathrm{sp}^{2[22]}$. likewise, at the same moment due to the graphitization phase, the distance between neighboring carbon atoms increases after the transition to the energy level, the phase change region density will decrease, which change the chemical properties of the material. So, based on the above polycrystalline diamond ablation threshold calculation, used $355 \mathrm{~nm}$ wavelength UV nanosecond laser with high energy density processed the PCD in the clean atmospheric environment, and analyzed the components before and after the laser machining.

The ESCALAB Xi + multi-function XPS was used to characterize the PCD samples before and after the laser micromachining. The excitation source is $\operatorname{ALK} \alpha$, during the operating condition of the vacuum pressure is maintained at $\sim 10^{-6} \mathrm{~Pa}$ with the $1 \mathrm{eV}$ acquisition step. In Fig 5 is show the full XPS energy survey spectrum of PCD samples before the ablation. It can be seen from the whole survey of the energy spectrum, there are two high peaks and some small 
peaks in the sample, the two high peaks show carbon $(\mathrm{C})$ and oxygen $(\mathrm{O})$ elements which is the most abundant element in the sample. The small peaks show the other elements which are in very less amount, it can't count. So, from the whole energy spectrum, it can be seen that the sample is mainly composed of Carbon and Oxygen.

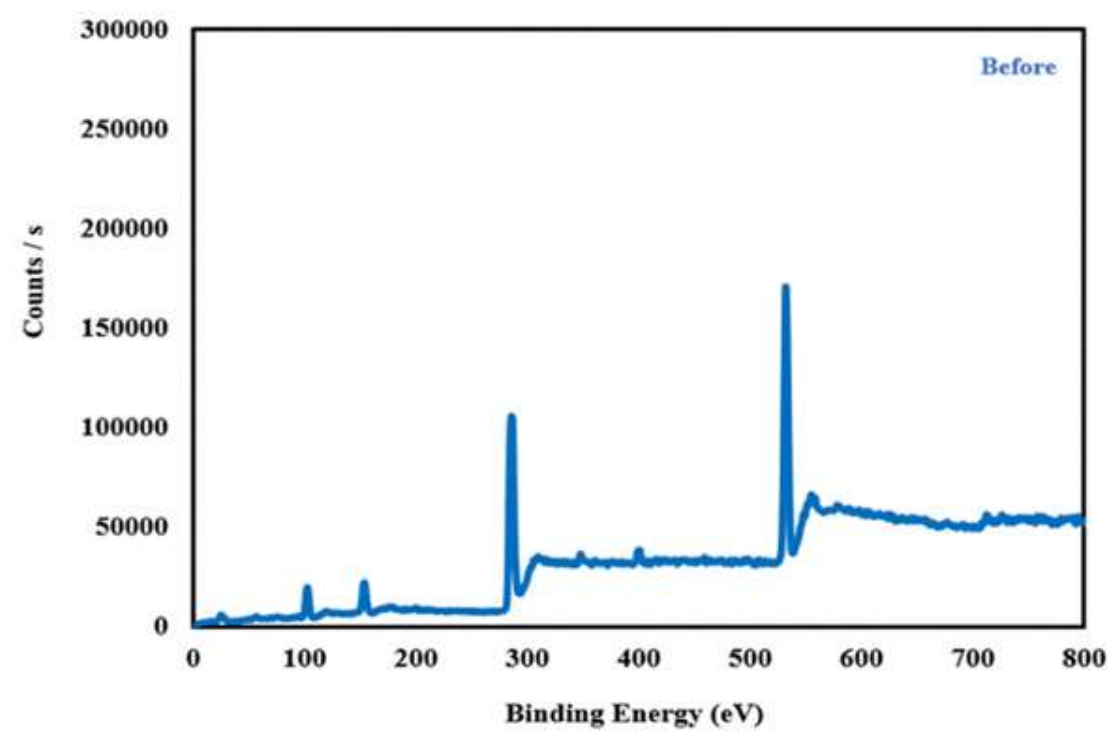

Figure 5 Polycrystalline diamond XPS energy survey spectrum

Fig 6 is a full energy survey spectrum of $\mathrm{C} 1 \mathrm{~s}$ of the sample and divided based on the original peak. C1s spectrum fitted in thermo Avantage software, divided into five characteristic peaks at 284.62, 285.63, 286.96, 288.24, and $289.90 \mathrm{eV}$, respectively. The strong peak at the binding energy of $286.96 \mathrm{eV}$ may be attributed to $\mathrm{C}-\mathrm{C} / \mathrm{Sp}^{2}$ diamond-like, it is $40.7 \%$ in content. At the binding energy $284.62 \mathrm{eV}$ belongs to the $\mathrm{C}=\mathrm{C}$ bond, content is $26.56 \%$, and at the binding energy $285.63 \mathrm{eV}$ attributed to $\mathrm{Sp}^{3}$ which belongs to diamond $18.6 \%$ content. At the binding energy $288.24 \mathrm{eV}$ attributed to satellite/C-O bond, content is $11.05 \%$, and at the high binding energy $289.90 \mathrm{eV}$ belongs to carbonyl $(\mathrm{C}=\mathrm{O})$ bond or $\mathrm{O}-\mathrm{C}=\mathrm{O}$ bond, which is $3.05 \%$ content. So, recognized that the element in $\mathrm{PCD}$ in the form of $\mathrm{C}-\mathrm{O}$, and there's no other composition. 


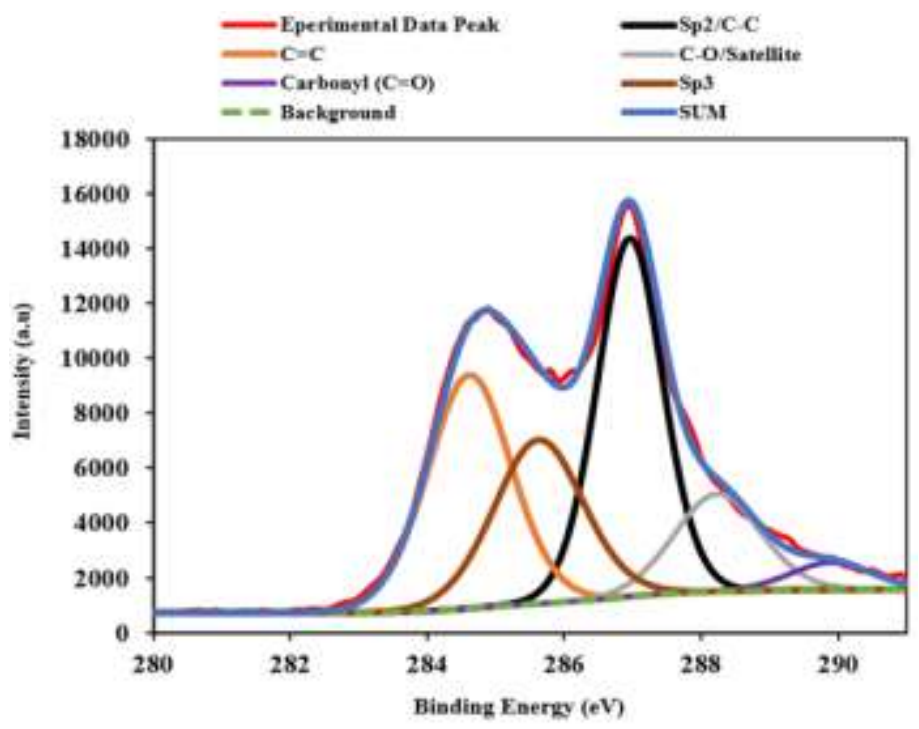

Figure 6 XPS energy survey spectrum before ablation

Fig 7 shows the full energy spectrum of O1s of the sample. It can be seen from the figure, the strong peak is located at the binding energy of $531.1 \mathrm{eV}$, and the peak shape is proportional to the original peak. The content of oxygen on the surface of the PCD may be due to the free oxygen in the atmosphere is adsorbed on the surface of the PCD or during the preparation processing of PCD during the liquid phase of organic solution. Besides, figure out that the carbon atom in the PCD has connected with oxygen atom chemically single bond or a double bond and has free oxygen adsorbed on the surface. The irradiation of nanosecond laser of PCD in the atmosphere, the air has a batch of oxygen, which makes the diamond carbon atoms in the absorption of energy after the transition and can be connected with the carbon atom and a large number of oxygen atoms. Hence, recognized that after irradiation, phase change of graphitization on the processed region of PCD, and the internal chemical modification (binding modes of atoms) also change. 


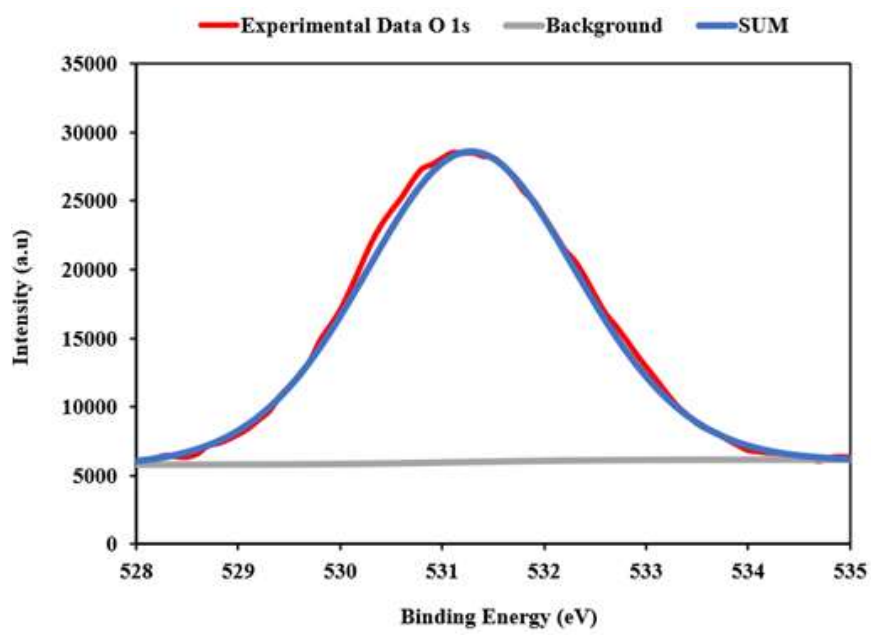

Figure 7 O1s XPS energy spectrum

The PCD sample was ablated by UV nanosecond laser and examined the post-processing area by XPS. Got full energy spectrum of PCD as shown in Fig 8. From the full energy spectrum, as can see that, the ablation area has the same two high peaks as achieved before the laser ablation. And there is no other element peaks and no transition phase of another element in the PCD after laser initiation in the atmospheric condition.

Fig 9 shows the C1s full energy survey spectrum of the ablated region of the PCD sample. It's divided based on the shape of the original peak and fitted into four characteristic peaks. The strong peak which located at the binding energy of $284.8 \mathrm{eV}$ attributed to the $\mathrm{C}=\mathrm{C} / \mathrm{Sp}^{2}$ bond $81.6 \%$ content, called graphite phase carbon. The characteristic peak at binding energy $286.4 \mathrm{eV}$ attributed to the satellite/C-O with the content of $11.2 \%$, and the characteristic peak at binding energy $288.4 \mathrm{eV}$ attributed to $\mathrm{O}-\mathrm{C}=\mathrm{O}$ bond, the content is $7.06 \%$. The characteristic peak located at the binding energy $284.8 \mathrm{eV}$ has a very big difference from the un-ablated region of the PCD sample, and this characteristic peak has not yet been described in the research and is not precisely defined. In this study, considering the processing environment, assume that $\mathrm{sp}^{3}$ changes to $\mathrm{sp}^{2}$ after the laser ablation.

Fig 10 shows the energy spectrum of O1s of the post-processing region. In the figure, as can be seen, that the characteristic strong peak is located at binding energy $531.1 \mathrm{eV}$. The shape of the peak is almost proportional to the SUM, which shows that the binding energy form of the oxygen atoms did not change before and after the laser ablation. 


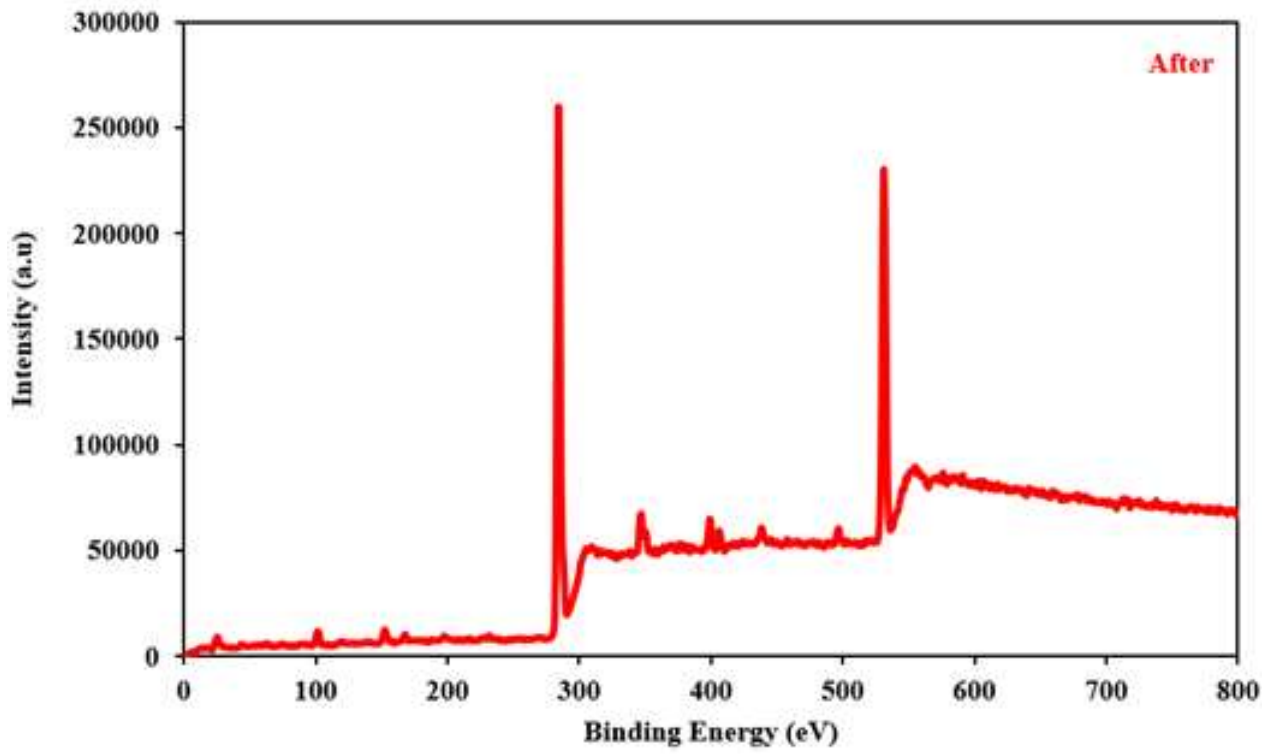

Figure 8 XPS energy survey spectrum after laser machining of polycrystalline diamond

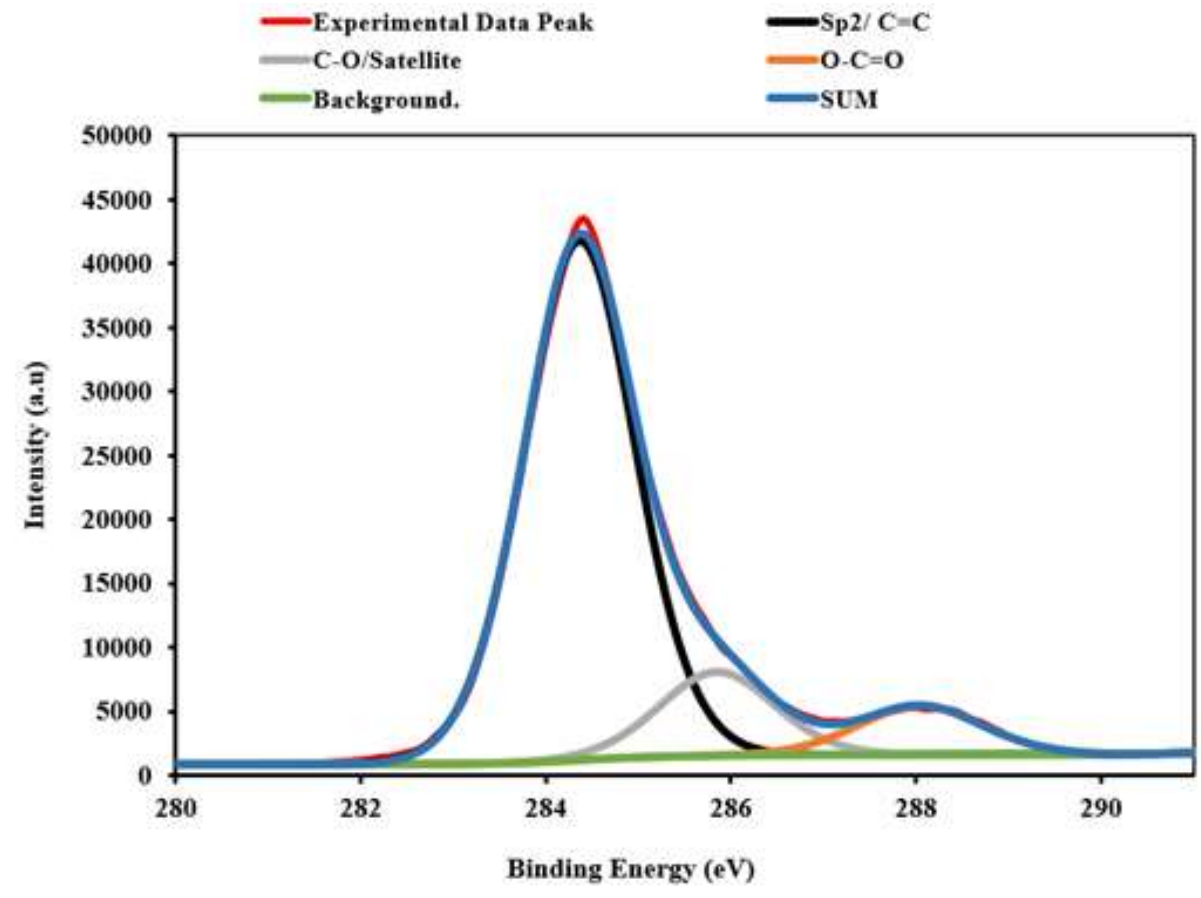

Figure $9 \mathrm{C}$ 1s XPS energy survey spectrum after the ablation 


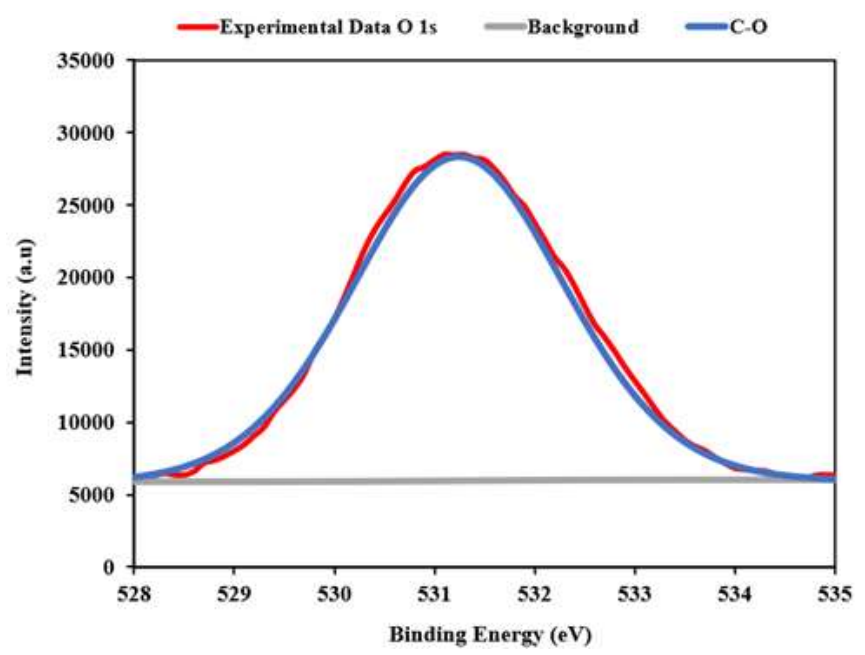

Figure 10 O 1s XPS energy spectrum

In Fig 11 the comparison between the XPS full energy survey spectrum before and after the UV nanosecond laser ablation. From the analysis of the energy spectrum, as can be seen, that the spectrum before and after the ablation is the same fundamentally, even the position of the two high peaks is not reduced. So, the results explain that the element in the PCD material before and after the laser ablation is fundamentally identical. But the content of carbon and oxygen elements in the laser-ablated region improved a lot. However, the contents of carbon elements increased significantly and the contents of oxygen elements a little bit increased at the same time. This means that after the laser ablation process the carbon element in PCD absorb oxygen from the atmosphere. And due to the laser ablation the $\mathrm{sp}^{3}$ changes to $\mathrm{sp}^{2}$ which is called the chemical modification (mechanism of the graphitization phase transition) of PCD after laser ablation. So, this result is in great agreement with the proposed hypothesis.

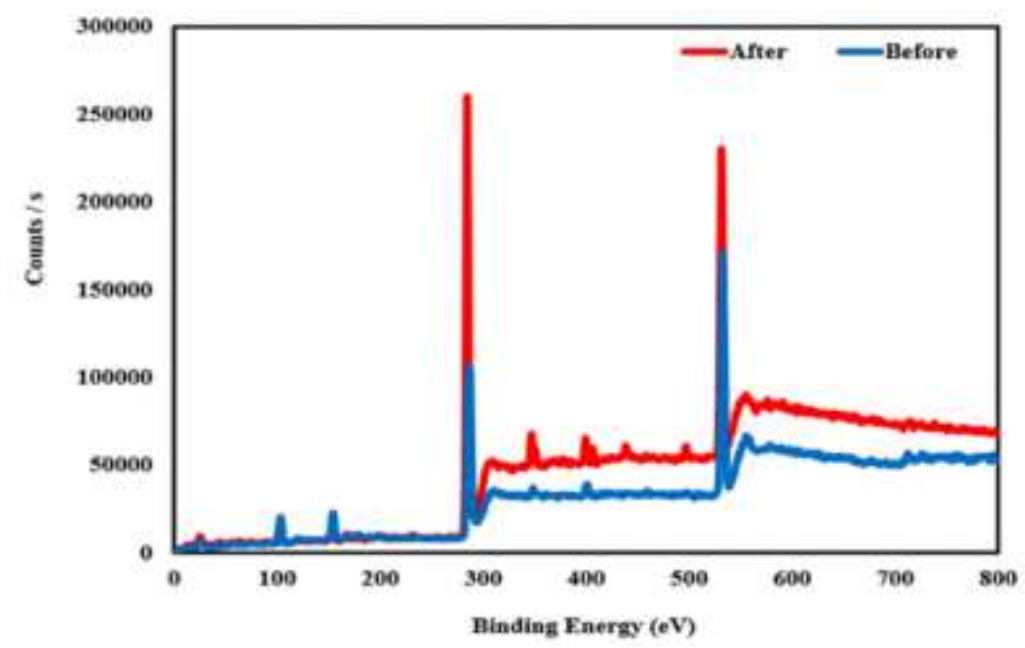

Figure 11 XPS energy survey spectrum before and after the laser ablation 


\section{Conclusion}

A UV nanosecond laser emitting at a wavelength of $355 \mathrm{~nm}$ has been used to determine the ablation threshold and chemical modification of PCD diamond. The onset of micropore formation is seen to take place at single pulse energy $3.7326 \mathrm{~J} / \mathrm{cm}^{2}$ with corresponding cumulative coefficient $S=0.985$. The etching rate as well as micropore formation at this energy fluence are observed as a subtle process and not a sudden removal of material. So, the measured value determined that the ablation threshold under the single-pulse laser energy density is accurate and reliable for PCD processing. Through XPS survey spectrum characterized the chemical modification before and after the UV nanosecond laser ablation of PCD and the modification of carbon atom structure convert from $\mathrm{sp}^{3}$ to $\mathrm{sp}^{2}$. These modifications disclose the mechanism of transition after laser irradiation of PCD to the graphitization phase.

To broaden the application of laser processing of diamond material, interaction mechanism between laser and PCD material, graphitic and rich nature of carbon laser micromachining sites has encouraged further investigations into the use of laser micromachining for diamond materials.

\section{Acknowledgments}

This work was supported by the Joint Fund for Equipment Pre-Research of the Ministry of Education (Young Talents Program) (6141A02033118), Key-Area Research and Development Program of Guangdong Province(2018B090905003), the Science and Technology Innovation Commission Shenzhen (JCYJ20180306170821261), National Natural Science Foundation of China (51735010, 51875450). All authors Thankfully acknowledge their support.

\section{References}

[1] KONOV V I. Laser in micro and nanoprocessing of diamond materials [J]. Laser \& Photonics Reviews, 2012, 6(6): 739-66.

[2] BRECHER C, EMONTS M, HERMANI J-P, et al. Laser roughing of PCD [J]. Physics Procedia, 2014, 56(1107-14.

[3] DOU J, CUI J, FANG X, et al. Theoretical and Experimental Study on Machining Rectangular Microgroove of Diamond by Femtosecond Laser [J]. Integrated Ferroelectrics, 2020, 208(1): 104-16.

[4] DUAN W, WANG K, DONG X, et al. Study on Machining of High Quality Micro Holes by Laser Trepan Drilling [J]. 2015,

[5] DOLD C, HENERICHS M, BOCHMANN L, et al. Comparison of ground and laser machined polycrystalline diamond (PCD) tools in cutting carbon fiber reinforced plastics (CFRP) for aircraft structures [J]. Procedia Cirp, 2012, 1(178-83.

[6] JIA Y H, LI J G, LU X J. Study on EDM machining technics of polycrystalline diamond cutting tool and PCD cutting tool's life; proceedings of the Advanced Materials Research, F, 2011 [C]. Trans Tech Publ. 
[7] WANG W, SONG H, LIAO K, et al. Water-assisted femtosecond laser drilling of 4H-SiC to eliminate cracks and surface material shedding [J]. The International Journal of Advanced Manufacturing Technology, 2020, 1-10.

[8] CUI J, YANG L, WANG Y. Simulation study of near-field enhancement on a laser-irradiated AFM metal probe [J]. Laser Physics, 2013, 23(7): 076003.

[9] CUI J, YANG L, XIE H, et al. New optical near-field nanolithography with optical fiber probe laser irradiating atomic force microscopy probe tip [J]. Integrated Ferroelectrics, 2016, 169(1): 124-32.

[10] XING Y, LIU L, HAO X, et al. Micro-channels machining on polycrystalline diamond by nanosecond laser [J]. Optics \& Laser Technology, 2018, 108(333-45.

[11] REN J, AHMED R, BUTT H. Finite element analysis of nanosecond pulsed laser ablation of various materials [J]. World Journal of Engineering, 2017,

[12] QI W. A study of the laser milling process for polycrystalline diamonds [D]; The University of New South Wales, 2013.

[13] EBERLE G, JEFIMOVS K, WEGENER K. Characterisation of thermal influences after laser processing polycrystalline diamond composites using long to ultrashort pulse durations [J]. Precision Engineering, 2015, 39(1624.

[14] KONONENKO V, GOLOLOBOV V, KONOV V. Latent laser-induced graphitization of diamond [J]. Applied Physics A, 2016, 122(3): 258.

[15] CHEN M, ZHANG F-L, OUYANG C-D, et al. Research advance on mechanism of micro machining of diamond materials by pulsed laser [J]. Superhard Mater Eng, 2014, $26(15$.

[16] PAN A, WANG W, LIU B, et al. Formation of high-spatial-frequency periodic surface structures on indium-tinoxide films using picosecond laser pulses [J]. Materials \& Design, 2017, 121(126-35.

[17] SAKURAI H, HE C, KONISHI K, et al. Effect of damage incubation in the laser grooving of sapphire [J]. Journal of Applied Physics, 2019, 125(17): 173109.

[18] MUSTAFA H, MATTHEWS D, RÖMER G. Investigation of the ultrashort pulsed laser processing of zinc at 515 nm: morphology, crystallography and ablation threshold [J]. Materials \& Design, 2019, 169(107675.

[19] ZHENG B, JIANG G, WANG W, et al. Ablation experiment and threshold calculation of titanium alloy irradiated by ultra-fast pulse laser [J]. AIP Advances, 2014, 4(3): 031310.

[20] CHENG C-W, CHEN J-K. Micro-and Nano-Structuring of Materials via Ultrashort Pulsed Laser Ablation [M]. Laser Ablation-From Fundamentals to Applications. IntechOpen. 2017.

[21] SUN Y, DOU J, XU M, et al. Research on the mechanism of micromachining of CVD diamond by femtosecond laser [J]. Ferroelectrics, 2019, 549(1): 266-75.

[22] CUI J, ZHANG J, HE X, et al. Investigating interfacial contact configuration and behavior of single-walled carbon nanotube-based nanodevice with atomistic simulations [J]. Journal of Nanoparticle Research, 2017, 19(3): 110. 
Figures

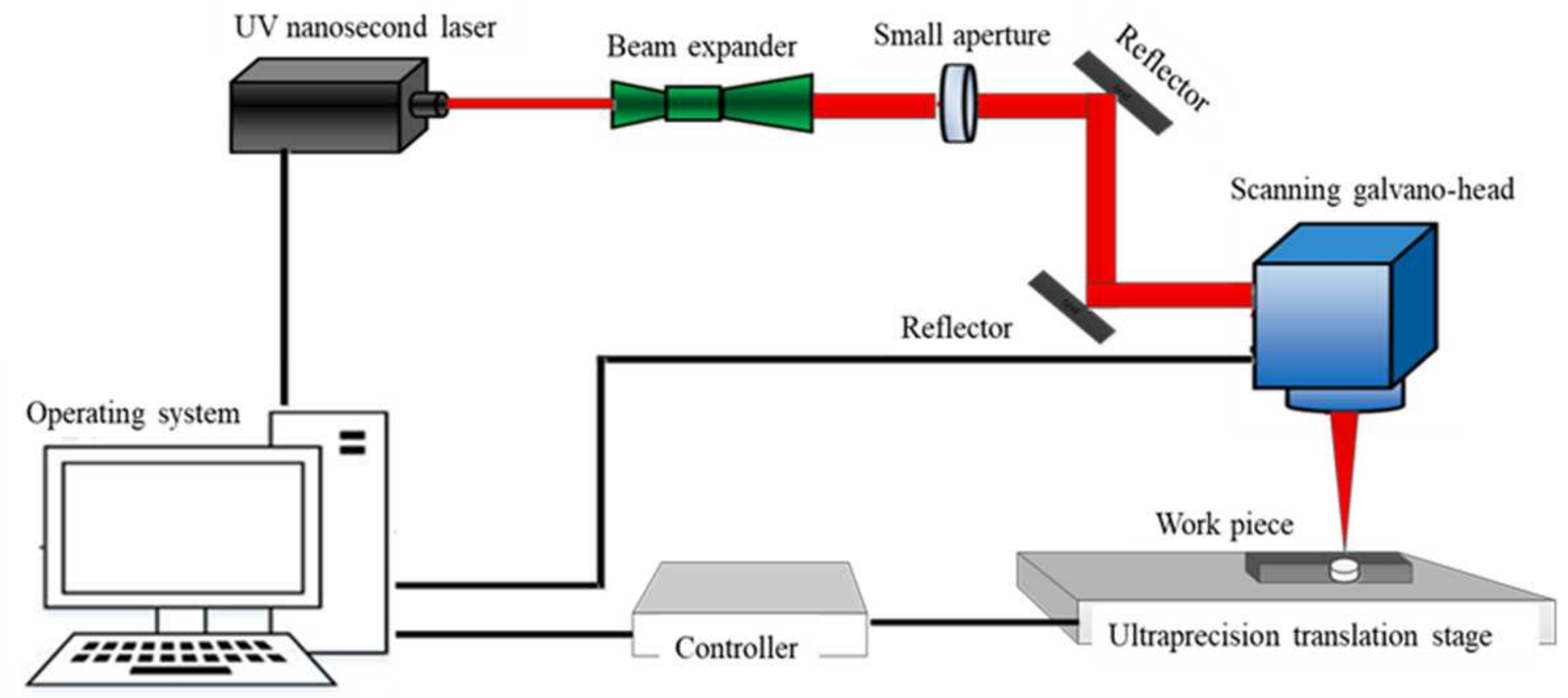

\section{Figure 1}

Schematic diagram of UV nanosecond laser machining system 


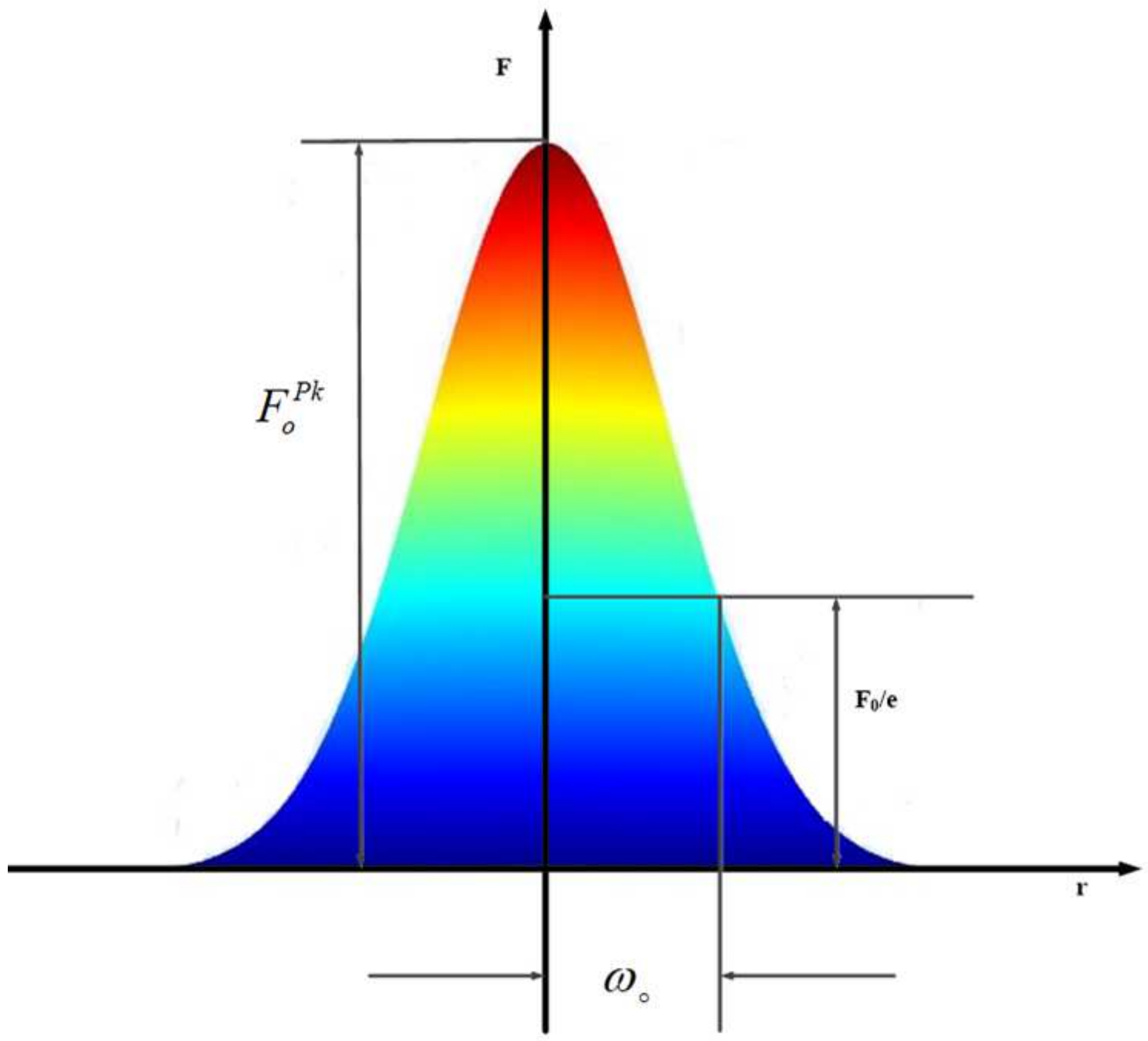

Figure 2

Energy distribution of gaussian beam 


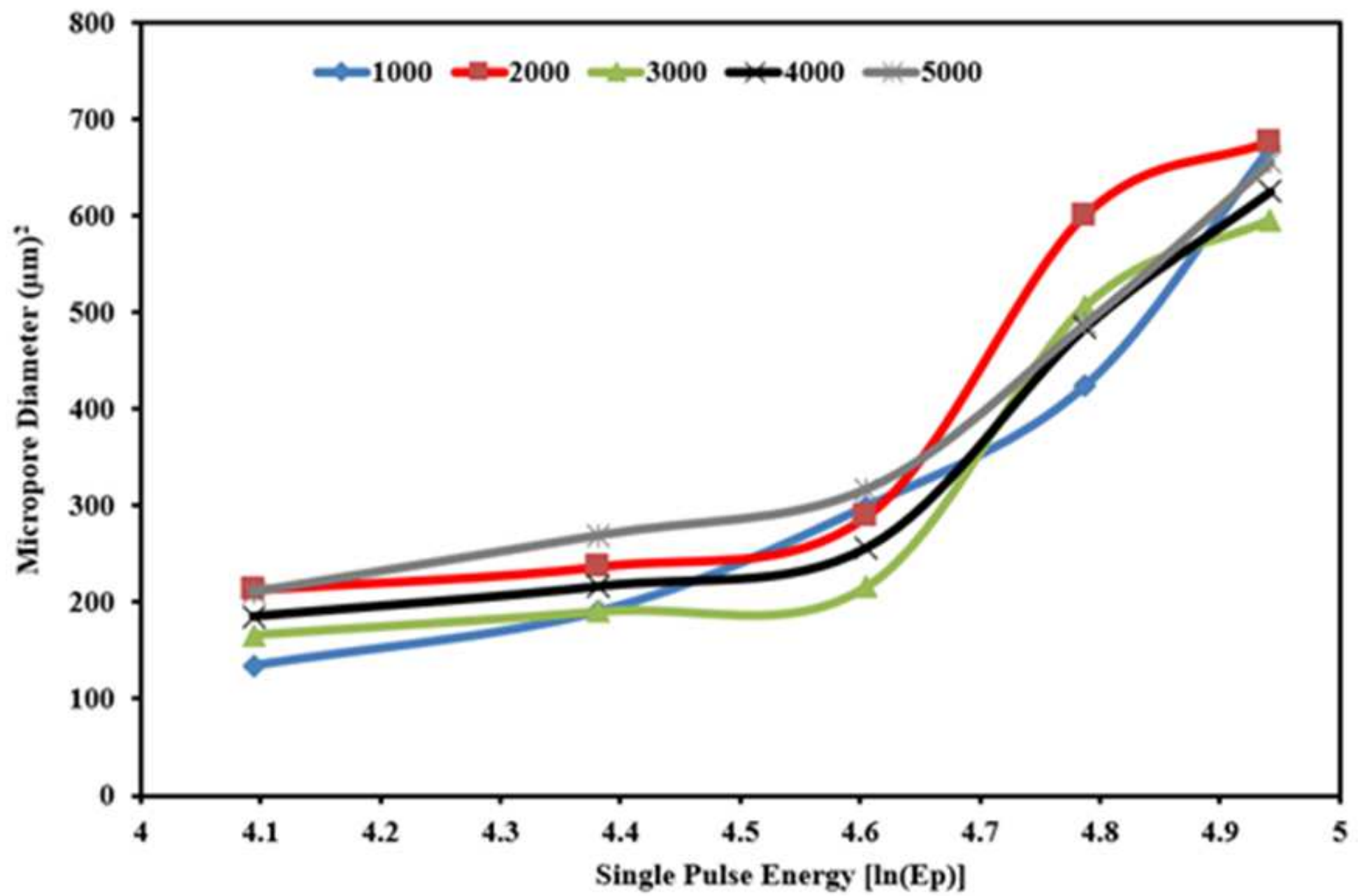

Figure 3

In (EP) and square of micropore diameter at numbers of pulse. 


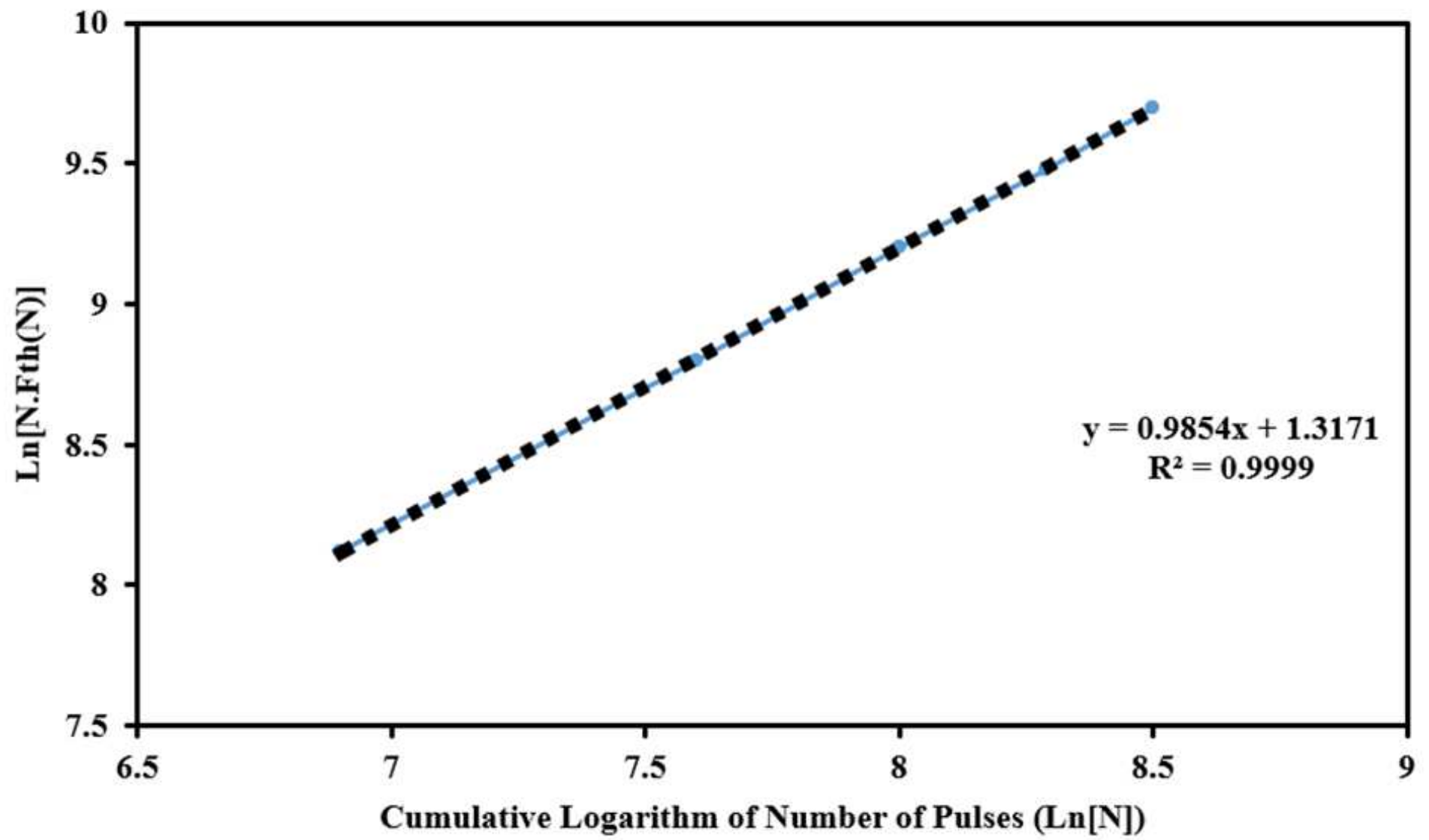

Figure 4

Cumulative effect of the number of pulses. 


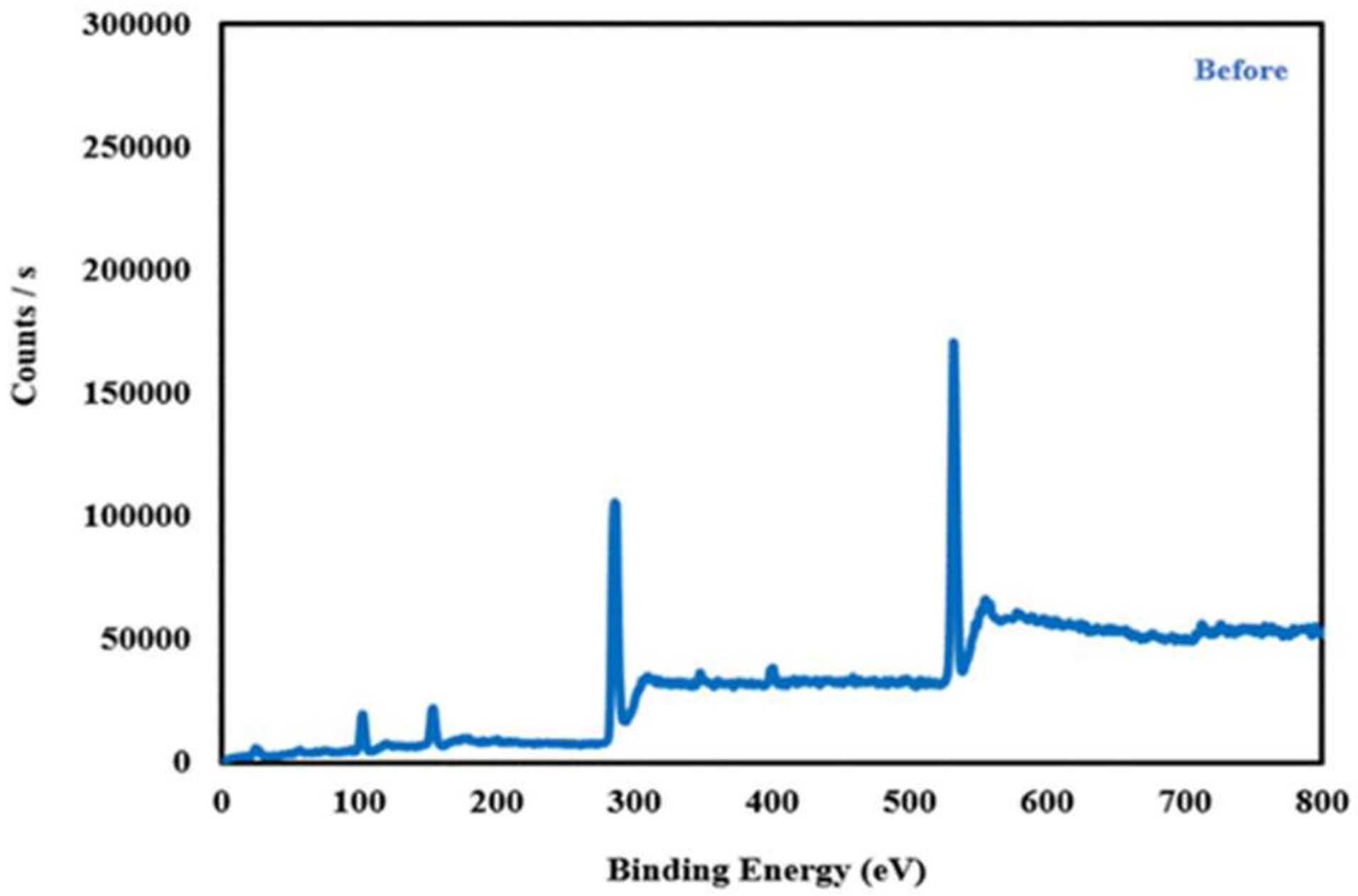

Figure 5

Polycrystalline diamond XPS energy survey spectrum 


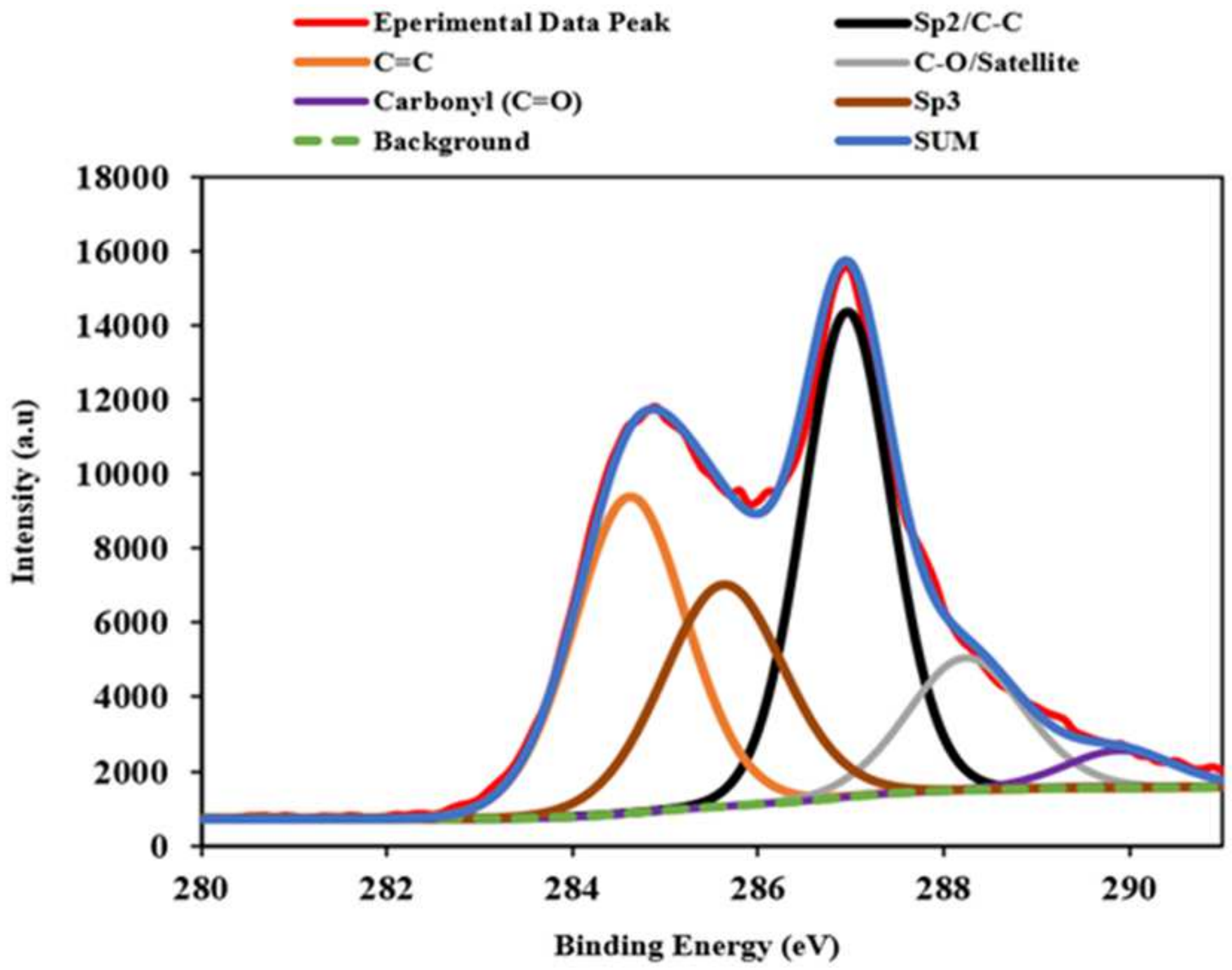

Figure 6

XPS energy survey spectrum before ablation 


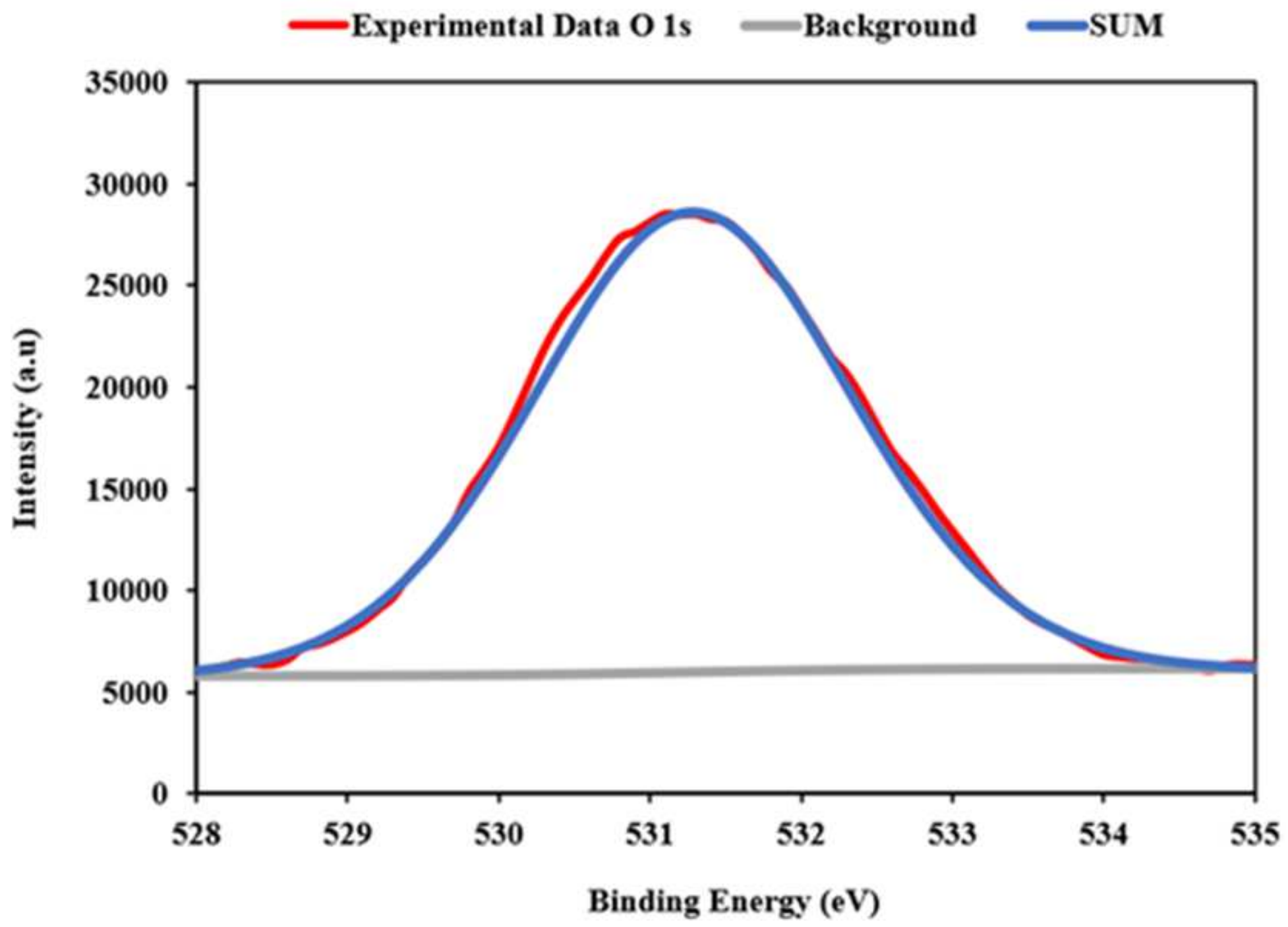

Figure 7

01s XPS energy spectrum 


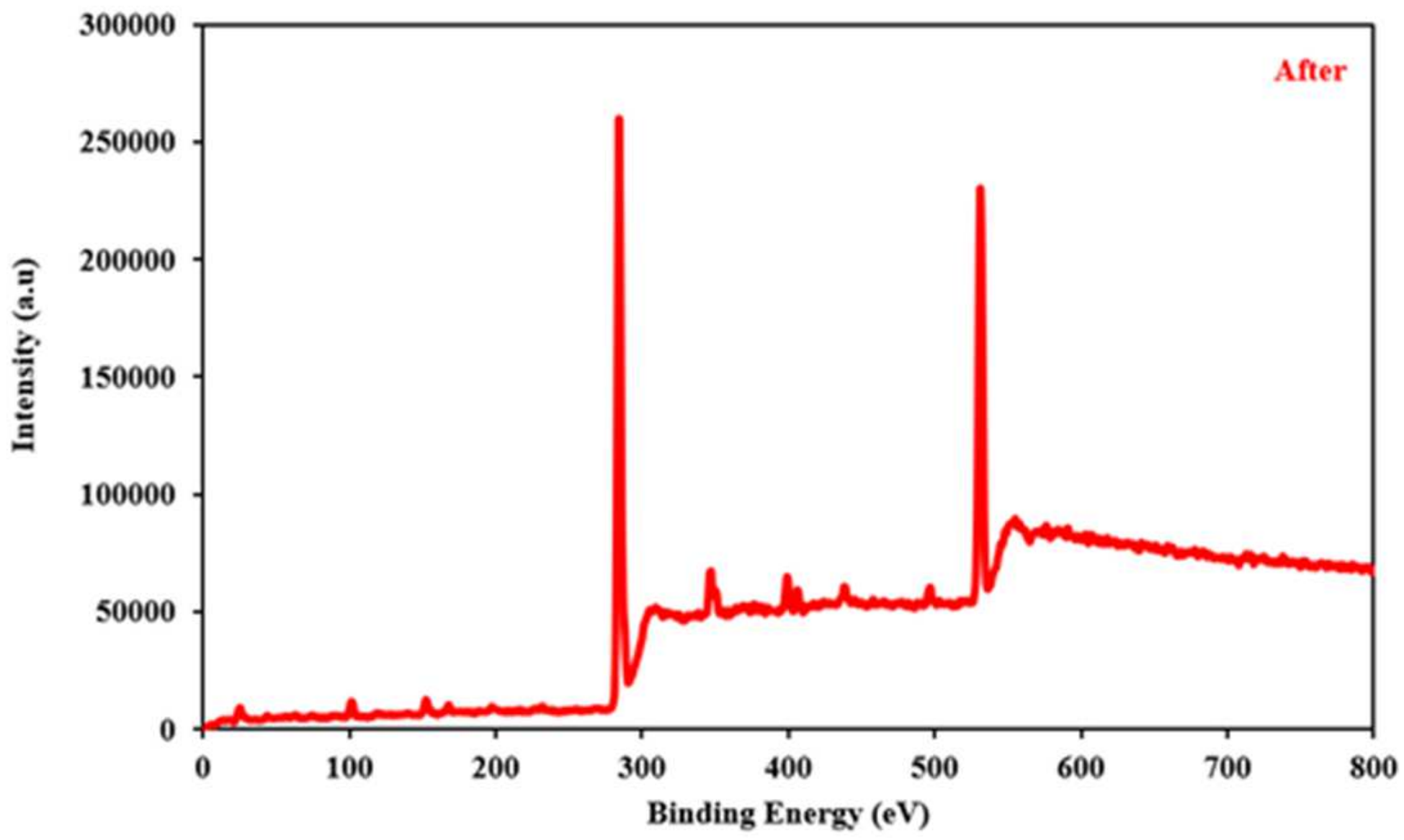

Figure 8

XPS energy survey spectrum after laser machining of polycrystalline diamond 


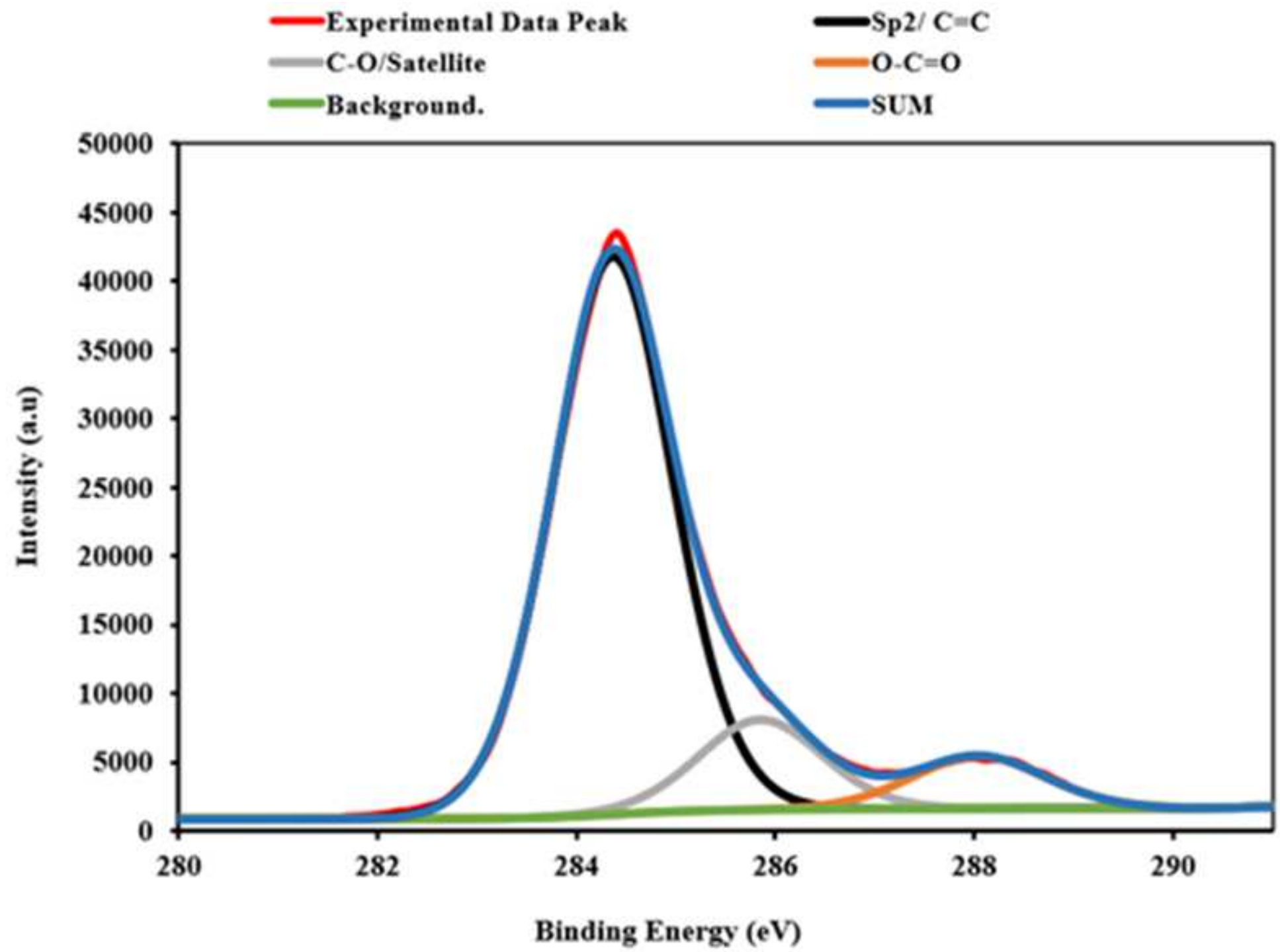

Figure 9

C 1s XPS energy survey spectrum after the ablation 


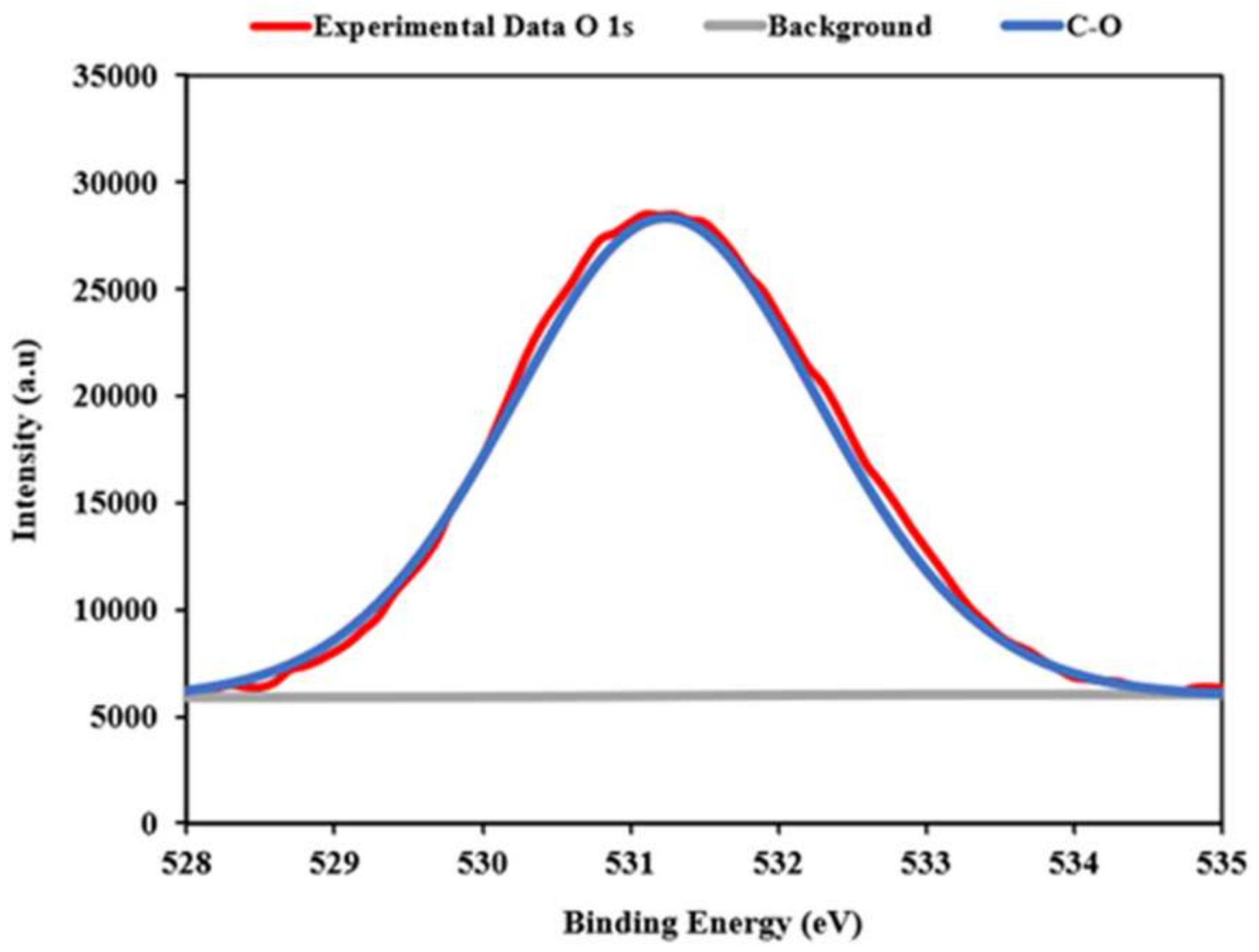

Figure 10

0 1s XPS energy spectrum 


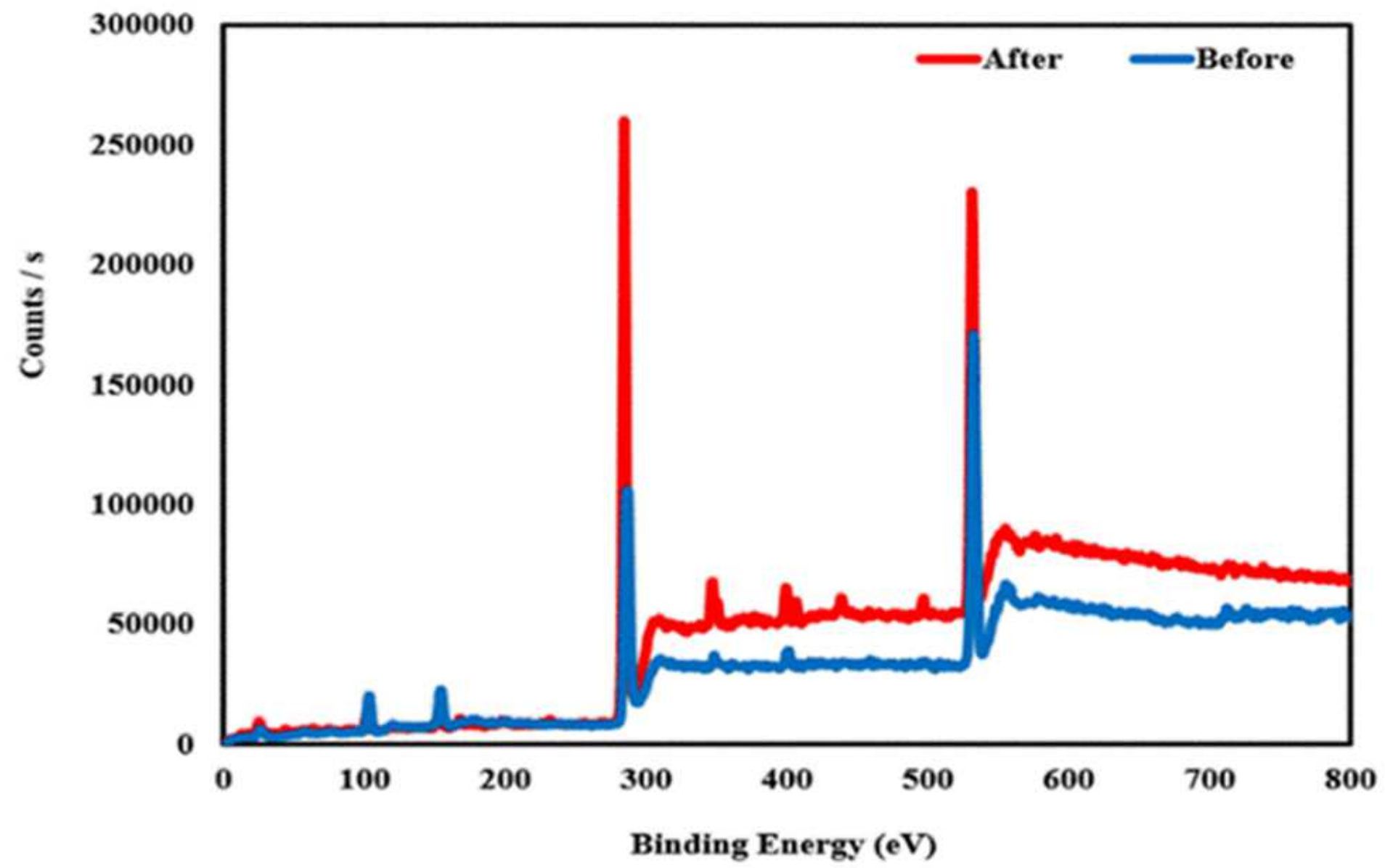

Figure 11

XPS energy survey spectrum before and after the laser ablation 